\title{
Un avenir de qualité
}

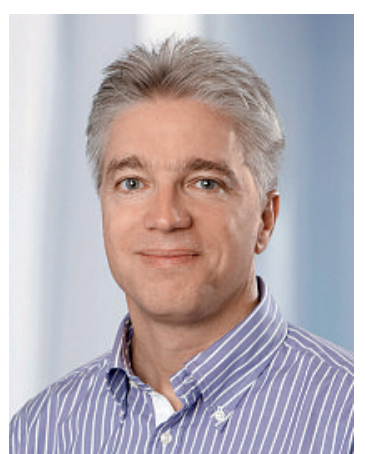

Connaissez-vous le concept d'«incentive-caused bias»? Derrière ce terme barbare se cache un phénomène que nous rencontrons en permanence dans les discussions sur la qualité en médecine. Il s'agit d'un mécanisme selon lequel certains incitatifs entraînent un changement de comportement qui va à l'encontre des intentions initiales. Rolf Dobelli l'a illustré de manière très parlante dans la Sonntagszeitung [1]. Dans le Vietnam du XIX ${ }^{\text {e }}$ siècle, une loi contre le fléau des rats a conduit à élever ces rongeurs plutôt qu'à les attraper - car il était bien plus facile de livrer des animaux élevés et d'encaisser la récompense, plutôt que de se fatiguer à en attraper.

Dans le domaine de la santé, nous serions également bien inspirés de mettre en place des incitatifs pertinents et mûrement réfléchis. Andrea Abraham [2] l'a particulièrement bien exprimé: avec l'entrée en vigueur de la LAMal, la qualité médicale a quitté le statut d'affaire interne aux sociétés médicales et peut maintenant faire l'objet de négociations entre différents acteurs - également étrangers à la médecine. Avec qui convient-il désormais de négocier et où nous situons-nous, notamment en ce qui concerne la question des incitatifs?

\section{Dans le domaine de la santé, nous serions également bien inspirés de mettre en place des incitatifs pertinents et mûrement réfléchis.}

L'année dernière, l'un des principaux partenaires de la FMH a sans aucun doute été l'Office fédéral de la santé publique (OFSP). En tant que représentants des médecins, nous avons pu nous investir comme nous le souhaitions et - après les changements à la tête du Département et de l'Office - mener enfin des discussions constructives. Le fruit de cette collaboration intensive, la mise en œuvre de la stratégie «Qualité» de la Confédération, se trouve à présent sur le bureau du Conseiller fédéral Didier Burkhalter et devrait encore donner pas mal de travail une fois la procédure de consultation terminée.

Les nouvelles en provenance des autres fronts sont moins réjouissantes. L'Association nationale pour le développement de qualité dans les hôpitaux et les cliniques (ANQ), qui s'est pourtant elle-même fixé le but et la mission de «coordonner et de piloter la garantie de la qualité [dans le domaine hospitalier] au niveau national» [3], a refusé d'intégrer la FMH dans son comité directeur. Notre demande, qui consistait à occuper l'un (!) des douze sièges de cet organe stratégique, a été rejetée. Le comité de l'ANQ, et le représentant de l'Association des hôpitaux $\mathrm{H}+$ en particulier, est d'avis que l'évaluation de la qualité dans le domaine hospitalier est du ressort exclusif des hôpitaux, des cantons et des assureurs. Le corps médical devrait tout au plus accompagner les évaluations de la qualité sur le plan du contenu au sein de groupes de travail, mais sans se prononcer sur la stratégie - une répartition des rôles inacceptable pour la FMH. Il est dès lors difficile de ne pas tomber dans le travers des «non-incentive-caused bias» et d'appeler au boycott de ces évaluations. Il est probable qu'avec la mise en œuvre de la stratégie «Qualité» de la Confédération, le rôle de l'ANQ sera redéfini, puisque les traitements ambulatoires et hospitaliers seront toujours plus étroitement liés et ne pourront plus être considérés comme deux entités séparées.

Les projets relatifs à la qualité qui font l'objet d'une collaboration avec santésuisse s'annoncent également difficiles. Préoccupé de sa réorganisation interne, qui dure depuis un certain temps déjà, l'organe faîtier des assureurs maladie a relégué la question de la qualité au second plan. Nos efforts visant à faire avancer des projets concrets sont tout simplement voués à l'échec en raison du manque d'engagement. Pour le moment, il nous semble donc plus productif de dialoguer directement avec les grands assureurs.

Par contre, la collaboration au sein de la nouvelle direction du Medical Board s'avère enrichissante et productive. Avec la Conférence suisse des directrices et directeurs cantonaux de la santé (CDS) et l'Académie Suisse des Sciences Médicales (ASSM), la FMH ouvre de nouvelles perspectives dans le domaine de l'HTA (Health Technology Assessment). Si en plus, conjointement avec Interpharma et santésuisse, nous parvenons à en élaborer les bases nécessaires, nous aurons accompli une avancée décisive dans le domaine HTA et pourrons rattraper notre retard sur d'autres pays. Mais nous devons là aussi être conscients du phénomène de $\mathrm{l}^{\prime}$ «incentive-caused bias» et tenter de concilier intention et incitatif. Au final, nous ne voulons en aucun cas encourager l'élevage de rats!

Dr Daniel Herren MHA,

Membre du Comité central de la FMH, Responsable du domaine $D D Q$

\section{Références}

1 Dobelli R. Warum Sie Ihren Anwalt nicht nach Aufwand bezahlen sollten. SonntagsZeitung du 26 décembre 2010.

2 Abraham A. Varianz als Norm. Bull Méd Suisses. 2010;91(49):1967-70.

3 Q-Info $\mathrm{n}^{\circ} 14$ de l'ANQ; décembre 2004. 